\title{
Combined flow-based imaging assessment of optimal cardiac resynchronization therapy pacing vector: a case report
}

\author{
A. R. Martiniello ${ }^{1 *}$, V. Bianchi ${ }^{1}$, G. Tonti ${ }^{2}$, C. Cioppa ${ }^{1}$, V. Tavoletta ${ }^{1}$, A. D'Onofrio ${ }^{1}$, V. M. Caso ${ }^{1}$, G. Pedrizzetti ${ }^{3}$ and \\ P. Caso ${ }^{1}$
}

\begin{abstract}
Background: There are still many pendent issues about the effective evaluation of cardiac resynchronization therapy impact on functional mitral regurgitation. In order to reduce the intrinsic difficulties of quantification of functional mitral regurgitation itself, an automatic quantification of real-time three-dimensional full-volume color Doppler transthoracic echocardiography was proposed as a new, rapid, and accurate method for the assessment of functional mitral regurgitation severity. Recent studies suggested that images of left ventricle flow by echo-particle imaging velocimetry could be a useful marker of synchrony. Echo-particle imaging velocimetry has shown that regional anomalies of synchrony/synergy of the left ventricle are related to the alteration, reduction, or suppression of the physiological intracavitary pressure gradients.
\end{abstract}

Case summary: We describe a case in which the two technologies are used in combination during acute echocardiographic optimization of left pacing vector in a 63-year-old man, Caucasian, who showed worsening heart failure symptoms a few days after an implant, and the effect of the device's optimization at 6-month follow-up.

Discussion: The degree of realignment of hemodynamic forces, with quantitative analysis of the orientation of blood flow momentum $(\varphi)$, can represent improvement of fluid dynamics synchrony of the left ventricle, and explain, with a new deterministic parameter, the effects of cardiac resynchronization therapy on functional mitral regurgitation. Realtime three-dimensional color flow Doppler quantification is feasible and accurate for measurement of mitral inflow, left ventricular outflow stroke volumes, and functional mitral regurgitation severity.

Conclusion: This clinical case offers an innovative and accurate approach for acute echocardiographic optimization of left pacing vector. It shows clinical utility of combined three-dimensional full-volume color Doppler transthoracic echocardiography/echo-particle imaging velocimetry assessment to increase response to cardiac resynchronization therapy, in terms of reduction of functional mitral regurgitation, improving fluid dynamics synchrony of the left ventricle.

Keywords: Mitral regurgitation, Three-dimensional echocardiography, Full-volume color Doppler echocardiography, Echo-PIV, Cardiac resynchronization therapy

\footnotetext{
* Correspondence: alfromar@alice.it

${ }^{1}$ Cardiology Division, Monaldi Hospital, AORN Ospedali dei Colli, Via

Leonardo Bianchi, 80131 Napoli, Italy

Full list of author information is available at the end of the article
}

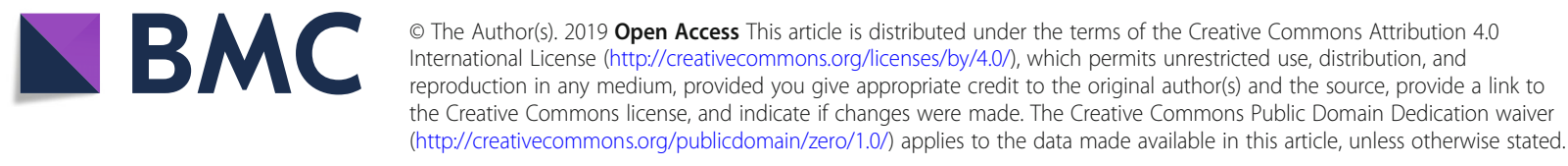




\section{Introduction}

The role of echocardiographic imaging is essential in the follow-up of patients after cardiac resynchronization therapy (CRT). Dysfunction of the device can be discovered by echo control with a multiparametric approach. This is the first case report in which automatic quantification of real-time three-dimensional full-volume color Doppler transthoracic echocardiography (FVCD) has been proposed as a new, rapid, and accurate method for the assessment of functional mitral regurgitation (FMR) severity pre-CRT and post-CRT, in combination with fluid dynamic approach using echo-particle imaging velocimetry (echo-PIV) technique.

Reduction in FMR is one of the mechanisms by which CRT exerts its beneficial effects. This innovative and combined approach can allow a more effective optimization of the biventricular device at follow-up. The quantification of mitral insufficiency during acute study can be associated with the quantitative analysis of the orientation of blood flow momentum $(\phi)$, which represents a new deterministic parameter of fluid dynamics synchrony. The combined approach can explain the effects of CRT on the realignment of hemodynamic forces involved in the pathophysiologic determinants of FMR. The short time of postprocessing during acute study would increase the cultural interaction between electrophysiologist and echocardiographer, with lower costs required than those necessary for invasive monitoring of cardiac performance that can be used only during implantation of the device.

\section{Case presentation}

We describe the case of a 63-year-old man, Caucasian, affected by non-ischemic dilated cardiomyopathy who did not drink alcohol, did not smoke tobacco, and did not have diabetes. He had an implantable cardioverter defibrillator implanted, in New York Heart Association (NYHA) IV class, and left bundle branch block (LBBB; QRS duration of $145 \mathrm{~ms}$ ). He was referred for CRT-D upgrade, awaiting cardiac transplantation, despite optimal medical therapy: b-Blockade, loop-diuretic, angiotensinconverting enzyme (ACE) inhibitor, K-sparing agent, and ivabradine. Standard clinical imaging protocol revealed a dilated left ventricle with an end-systolic volume (ESV) of $380 \mathrm{ml}$, an ejection fraction (EF) of $4.8 \%$ as measured by the modified Simpson's method, and severe FMR, assessed by qualitative estimation with two-dimensional color flow Doppler approach, showing a very large central jet and reaching the posterior wall of the left atrium (see Fig. 1 and Additional file 1: Video S1).

He underwent the implant of a CRT-D device with a quadripolar left ventricular (LV) lead placed in the posterolateral branch of the coronary sinus. After recording the right ventricle (RV)-to-LV electrical delay at each of

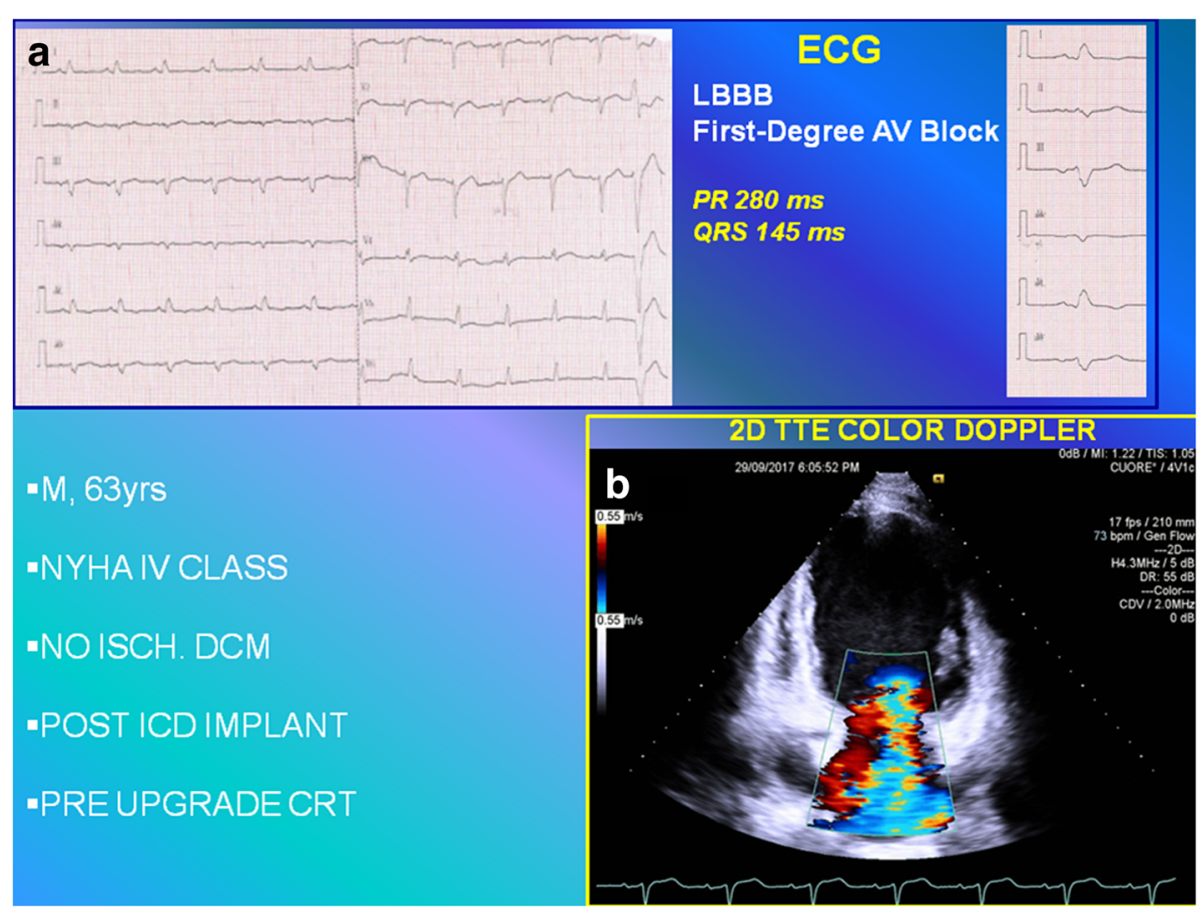

Fig. 1 a Twelve-lead electrocardiogram showing the wide native QRS complex (145 ms) with first-degree atrioventricular block (PR 280 ms). b Two-dimensional color transthoracic echocardiogram apical four-chamber view. AV atrioventricular, CRT cardiac resynchronization therapy, ECG electrocardiogram, ICD implantable cardioverter defibrillator, LBBB left bundle branch block, M male, NO ISCH. DCM non-isChemic dilated cardiomyopathy, NYHA New York Heart Association, TTE transthoracic echocardiogram 
the four LV rings, we chose the A1 unipolar vector for LV pacing (greatest electrical delay $80 \mathrm{~ms}$ ).

At 13-day post-implant follow-up, he showed worsening heart failure (HF) symptoms and only A2 unipolar LV vector configuration, with interventricular (VV) interval of $0 \mathrm{~ms}$, was suitable for simultaneous biventricular activation (Fig. 2).

Echo-PIV was then used, during the acute study with contrast agent bubbles, to evaluate the orientation and relative magnitude of blood-induced intraventricular forces in correspondence of different pacing settings.

Without pacing stimulation (CRT OFF, Fig. 3a, and Additional file 2: Video S2) the intraventricular flow was dominated by rotation without evident inflow-outflow dynamics. As a result the intraventricular forces were predominantly transverse and not aligned along the LV axis (Fig. $3 \mathrm{a}_{1}$ ) as quantified by the large value of their mean angle $\phi\left(\phi=55.6^{\circ}\right.$, this angle ranges from $0^{\circ}$, when forces are aligned with the LV axis, to $90^{\circ}$ ). A first setting option (CRT ON, VV delay 0 ms, Fig. 3b and Additional file 3: Video S3) changed the orientation of intraventricular forces (Fig. $\left.3 \mathrm{~b}_{1}\right)$ reducing the angle $\left(\phi=45^{\circ}\right)$, and increasing the delay (CRT ON, VV delay $-30 \mathrm{~ms}$, Fig. 3c and Additional file 4: Video S4) improved the alignment (Fig. $\left.3 c_{1}\right)$ reducing the angle $\left(\phi=40.3^{\circ}\right)$. Eventually, the sequential biventricular activation with delay $-50 \mathrm{~ms}$ (Fig. 3d and Additional file 5: Video S5) provided the best alignment of intraventricular forces (Fig. $3 \mathrm{~d}_{1}, \phi=38.8^{\circ}$ ).

No reduction of FMR by three-dimensional FVCD, during the same acute study with shutdown versus reactivation of device, was demonstrated, as shown in Figure 4 and by comparing Additional file 6: Video S6 and Additional file 7: Video S7.
The data acquisition time, by three-chamber apical view, for each three-dimensional color Doppler data set was approximately 5 seconds, and it took less than 3 minutes to analyze the average regurgitation volume, with automated anatomy detection of the LV endocardial border, mitral annulus (MA), LV outflow (LVOT), and placement of three-dimensional hemispheric flow sampling planes in the MA and LVOT. The software of three-dimensional FVCD computed the flow volumes as the area under the curve of both the MA and LVOT flow in three cardiac cycles, and FMR volume was calculated by subtracting LVOT stroke volume from MA stroke volume.

\section{Results at 6-month follow-up}

Our patient showed an improvement of NYHA class (III versus IV) and LV EF (26.6\% versus $4.8 \%$ ). Significant reduction of ESV ( $288 \mathrm{ml}$ versus $380 \mathrm{ml}$ ) and persistent improvement of diastolic function were obtained. The regularized function is noticeable in Additional file 8: Video S8 (to be compared with Additional file 1: Video S1) and it is summarized in Fig. 5. At follow-up, a significant reduction of FMR (mean value regurgitant volume, $42.2 \mathrm{ml}$ versus $65.3 \mathrm{ml}$ ) was estimated (Fig. 6, Additional file 9: Video S9, Table 1).

The intraventricular forces estimated by echo-PIV were still partially dominated by the longitudinal path of pressure gradient (Fig. 7 and Additional file 10: Video S10) with $\phi=43.1^{\circ}$.

\section{Discussion}

The availability of pacing configurations offered by quadripolar LV leads could improve patients' response to CRT, in terms of reduction of FMR, by improving

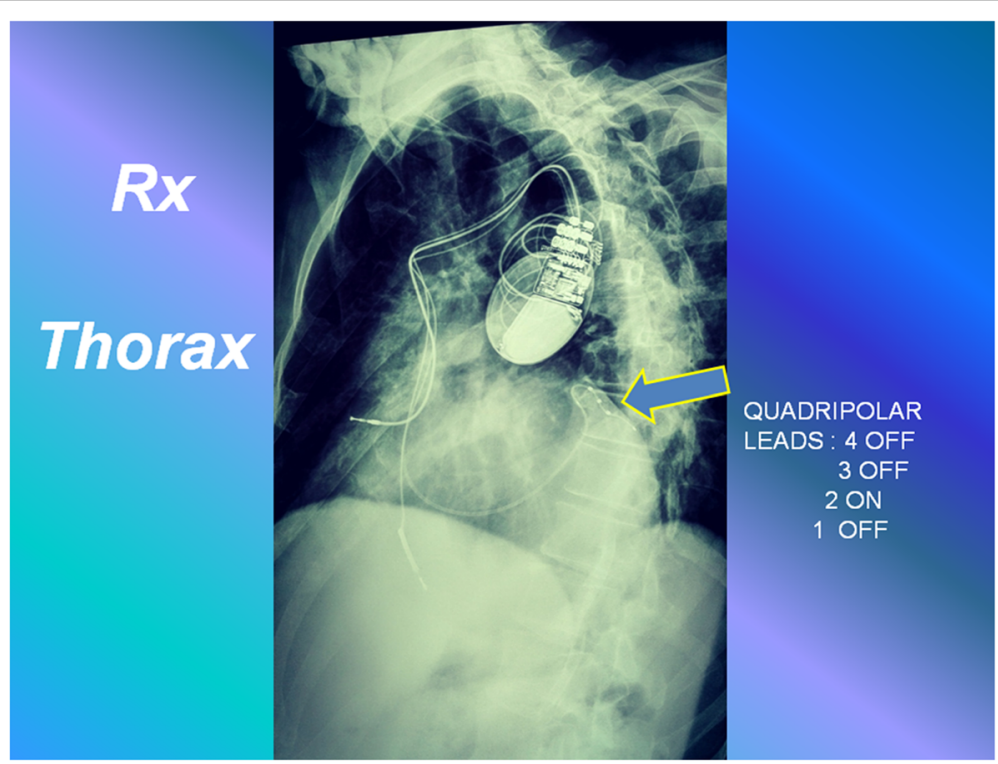

Fig. 2 Left anterior oblique chest X-ray view displaying the left ventricular quadripolar lead (arrow) 


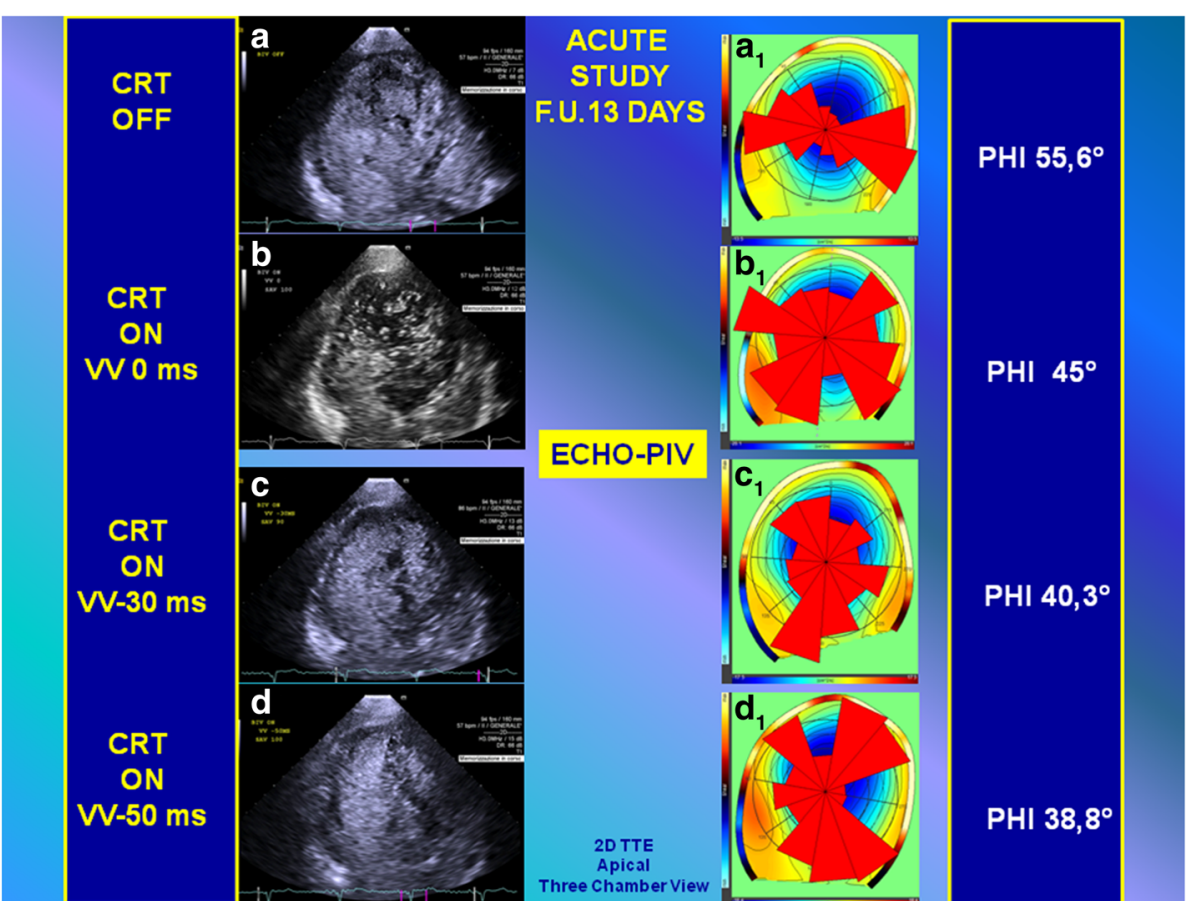

Fig. 3 Quantitative analysis (to compare with the results of analysis represented in Fig. 7) by echo-particle imaging velocimetry of the orientation angle $(\varphi)$ of the global hemodynamic forces exchanged between blood and surrounding tissues during acute study (postcardiac resynchronization therapy 13-day follow-up). Left: Two-dimensional transthoracic echocardiogram high-temporal resolution contrast echocardiographies are performed by three-chamber apical view approach during acute study of different setting (a, b, c, d). Right: Changes in electrical activation settings modify the orientation of intraventricular forces during acute study. The intraventricular forces were predominantly transverse and not aligned along the LVaxis $\left(\mathbf{a}_{1}\right)$ as quantified by the large value of their mean angle $\varphi\left(\varphi=55.6^{\circ}\right)$. A first setting option (CRT ON, W delay 0 ms) changed the orientation of intraventricular forces $\left(\mathbf{b}_{1}\right)$ reducing the angle $\left(\varphi=45^{\circ}\right)$, and increasing the delay (CRT ON, W delay $-30 \mathrm{~ms})$ improved the alignment $\left(\mathbf{c}_{\mathbf{1}}\right)$ reducing the angle $\left(\varphi=40.3^{\circ}\right)$. The sequential biventricular activation with delay $-50 \mathrm{~ms}\left(\mathbf{d}_{\mathbf{1}}\right)$ provided the best alignment of intraventricular forces $\left(\varphi=38.8^{\circ}\right)$. CRT cardiac resynchronization therapy, F.U. follow-up, PIV particle imaging velocimetry, W interventricular, TTE transthoracic echocardiogram

cardiac synchrony; however, the selection of an optimal setting remains a challenge, and the correct quantitative evaluation of FMR suffers from well-known pitfalls and systematic inaccuracy. The need for shape assumptions and the inability to account for the dynamic regurgitant orifices are technical limitations to two-dimensional proximal isovelocity surface area (PISA)-based effective regurgitant orifice area (EROA) and regurgitant volume measurements, especially in patients with FMR where the regurgitant orifice is thought to be largest at the beginning and end of systole, and smallest in the middle. Pulsed wave (PW) Doppler-based flow quantification techniques with two-dimensional transthoracic echocardiogram have been used to measure mitral inflow and aortic stroke volumes. This information is also used in computing flow-derived valve area in stenotic valve disease and regurgitant volume and fraction in valvular regurgitation. We know that the assumption of circular geometry of LVOT is erroneous and affects the calculation of aortic valve area by continuity equation, and the effect of spatially non-uniform flow on the continuity equation has not been clearly evaluated. Small errors in measurements of LVOT or mitral annular diameter are squared in the computation of stroke volume, and may lead to large differences of results during acute study. Moreover, PW Doppler of LVOT flow and MA flow are obtained in a separate echocardiographic windows, the timing of the measurements are different and can introduce error. Current three-dimensional color Doppler-based methods require the operator to trace the area of color flow and use electrocardiographically gated three-dimensional echo over an acquisition of more than seven complete cardiac cycles. Furthermore, as arrhythmias and respiratory movement generate a stitching artifact, it is necessary to optimize acquisition via sinus rhythm gating and breath holding. At the same time, a novel real-time three-dimensional FVCD, based on instantaneous acquisition of a single cardiac cycle, has been reported as an innovative method to assess FMR [1] without significant manual interaction with the data post-processing. FMR quantification with three-dimensional FVCD showed better correlation and agreement than conventional 


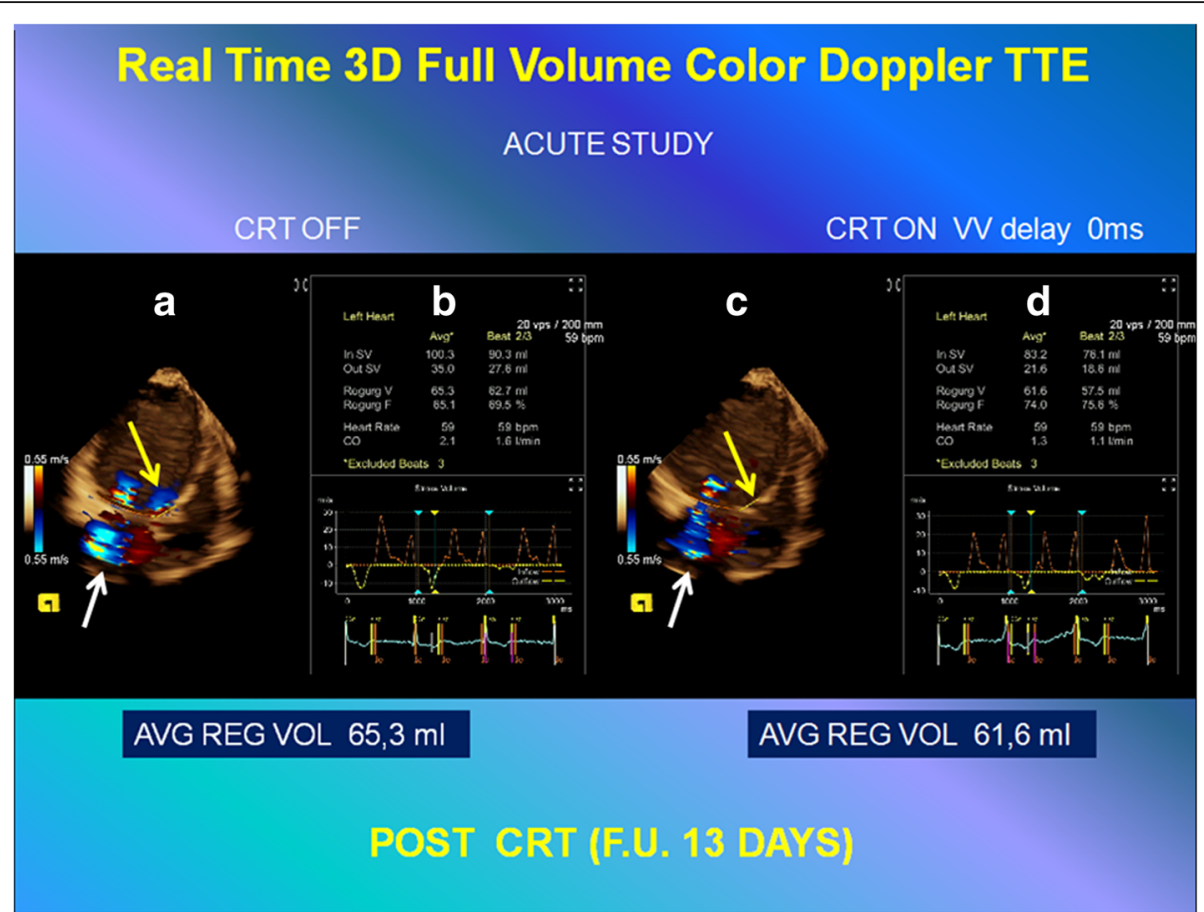

Fig. 4 Quantitative analysis (to compare with the results of analysis represented in Fig. 6), of functional mitral regurgitation by three-dimensional fullvolume color Doppler transthoracic echocardiography: acute study (post-cardiac resynchronization therapy 13-day follow-up). Left: Setting cardiac resynchronization therapy OFF. Right: Setting cardiac resynchronization therapy ON with interventricular delay 0 ms. a, c Automated anatomy detection of the left ventricular endocardial border, mitral annulus, left ventricular outflow, and placement of three-dimensional hemispheric flow sampling planes in the mitral annulus (white arrow) and left ventricular outflow (yellow arrow). b, d Flow-time curve derived from automatic flow volume aggregation at each frame in the cardiac cycle and the automated calculation of the mitral (In SV), aortic (Out SV) stroke volume, regurgitant volume, regurgitation fraction (RF), and cardiac output (CO), with mean value, in three cardiac cycles, respectively. AVG mean value, CRT cardiac resynchronization therapy, F.U. follow-up, REG VOL regurgitant volume, TTE transthoracic echocardiogram, W interventricular

two-dimensional methods. FMR was underestimated by two-dimensional methods, especially in multijet and dilated left ventricle. Multijet mitral regurgitation demonstrated a higher risk of discrepancy for the identification of surgical candidate, regardless of mitral regurgitation etiology [2]. This novel three-dimensional color Doppler flow quantification method based on instantaneous acquisition of a single cardiac cycle using a 4Z1c Matrix Array Transducer (Siemens Medical Solutions), which has 1728 elements, was used with the SC2000 system to obtain real-time non-gated three-dimensional volume and volume color Doppler images for this study. The three-dimensional data acquired included flow velocity information via color Doppler data, which have been made available by improvements in transducer and post-processing software technologies, to calculate mitral average regurgitation volume and stroke volumes semi-automatically from three-dimensional color Doppler data acquired at the mitral valve (MV) annulus and LVOT, with a wide-angle pyramidal volume. Previous two-dimensional and three-dimensional methods were not able to calculate stroke volumes at the MV and
LVOT simultaneously. The data acquisition time was approximately 5 seconds for each three-dimensional color Doppler data set, and it took less than 3 minutes to analyze each stroke volume. The hemispheric program for sampling planes needed minimal manual adjustment by the operator for accurate analysis of cardiac output. It was demonstrated that measurement of flow volume through the MV by three-dimensional color Doppler echocardiography was correlated and agreed well with cardiac magnetic resonance imaging [1-3]. Gruner et al. have shown that the quantification of FMR before and after percutaneous MV repair by three-dimensional FVCD was comparable to the integrative visual assessment and more reliable than the PW Doppler method [4]. Kato et al. have described the accuracy and feasibility of computing mitral, aortic, tricuspid, and pulmonic stroke volumes using three-dimensional FVCD in children, to compute valve areas and regurgitant volumes [5]. These authors have concluded that this technique potentially provides a non-invasive alternative to historically invasively acquired hemodynamic data and to cardiac magnetic resonance imaging [5]. Intracardiac flow is a 


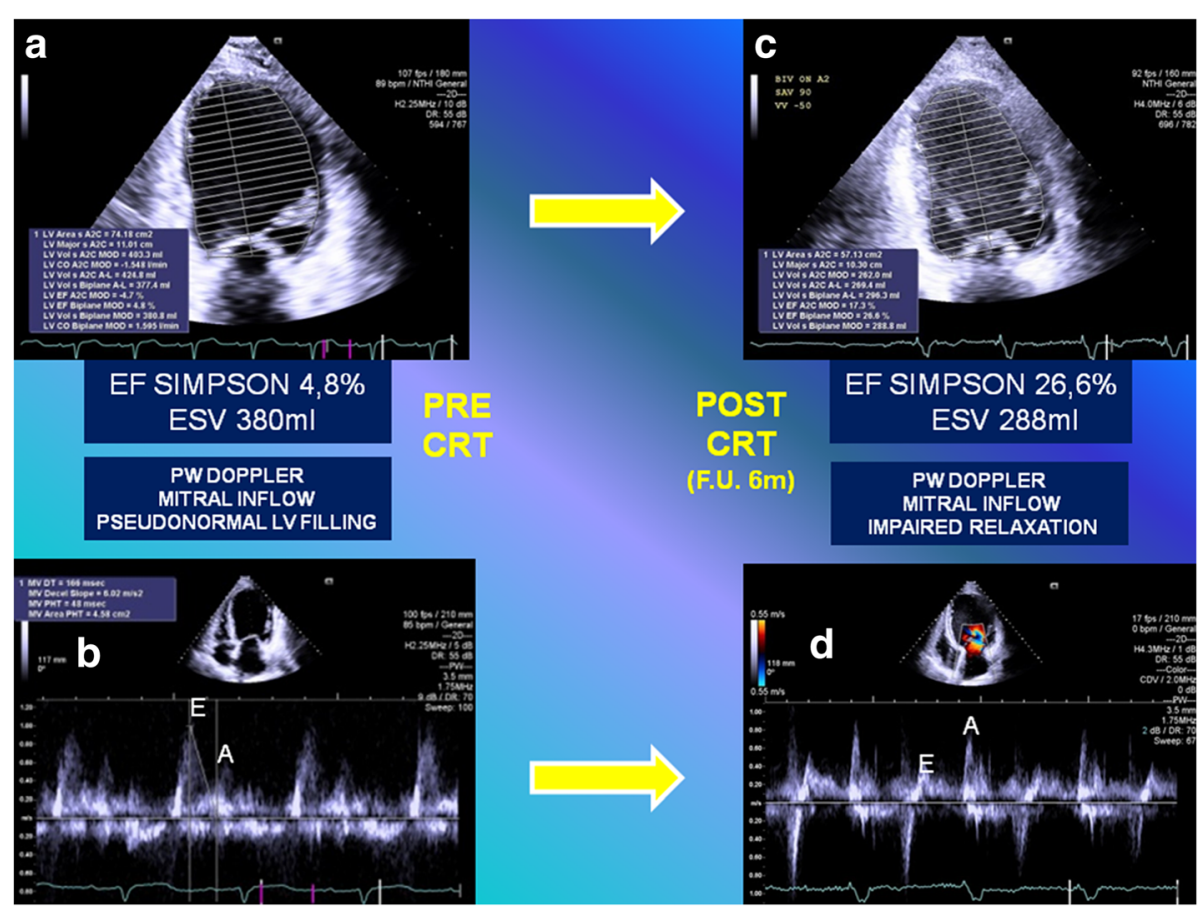

Fig. 5 Left side: a Dilated left ventricle with an end-systolic volume of $380 \mathrm{ml}$, and an ejection fraction of $4.8 \%$ as was measured by the modified Simpson's method pre-implant. b Pulsed wave Doppler mitral inflow pattern showing advanced diastolic dysfunction (deceleration time (DT) of early filling velocity, $165 \mathrm{~ms}$ ). Right side: c Dilated left ventricle with reduced end-systolic volume of $288 \mathrm{ml}$ and improved ejection fraction of 26.6\% as was measured by the modified Simpson's method post-implant at 6-month follow-up. d Pulsed wave Doppler mitral inflow pattern showing impaired relaxation. CRT cardiac resynchronization therapy, EF ejection fraction, ESV end-systolic volume, F.U. follow-up, LV left ventricle, PW pulsed wave

key physiological event that, in most instances, mediates the clinical consequences of anatomical perturbations. Three-dimensional echo is uniquely placed to overcome some of the well-known limitations of two-dimensional echo for flow quantification [6]. This is the first case report where automatic quantification of real-time threedimensional FVCD has been proposed as a new, rapid, and accurate method for the assessment of FMR severity pre-CRT and post-CRT in combination with fluid dynamic echo-PIV approach.

Echo-PIV is an optical method where the contrast agent bubbles are tracked from one frame to the next to calculate the instantaneous blood velocity field. EchoPIV has shown that regional anomalies of synchrony of the LV are related to the alteration of the physiological intracavitary pressure gradients that deviate from their natural longitudinal orientation $[7,8]$. This deviation can be assessed by quantitative analysis of the orientation angle $(\phi)$ of the global hemodynamic forces exchanged between blood and surrounding tissues. Many echocardiographic studies have demonstrated how CRT can contrast all the pathophysiologic determinants of FMR by minimizing LV dyssynchrony due to the following: increasing "closing forces" (global synchronization), reducing "tethering forces" (local synchronization), reshaping annular geometry and function (local synchronization), and correcting diastolic mitral regurgitation (atrioventricular synchronization). The role for routine VV delay optimization post-CRT is not clear. Most studies have shown that a majority of patients have optimal VV intervals that are within a range of \pm 20 milliseconds. Simultaneous biventricular pacing or pre-excitation of LV most often remains a challenge. VV delay optimization is generally performed by changing the VV sequence, starting with the LV being activated before the RV, and then stepwise lengthening or shortening of the VV interval (for example, with intervals of 20 milliseconds) and measuring the highest aortic time-velocity integral (Ao IVT), but this simplified echocardiographic approach is susceptible to inter-observer variability because there is a significant manual interaction with the data post-processing. Furthermore, the availability of pacing configurations offered by quadripolar left ventricle leads could improve a patient's response; however, selection of an optimal setting remains a challenge. Recent studies suggested that images of LV flow by echo-PIV could be a useful marker of synchrony [9]. The vortical hydrodynamic forces and their cytomechanical consequences by mechanosensing and mechanotransduction 


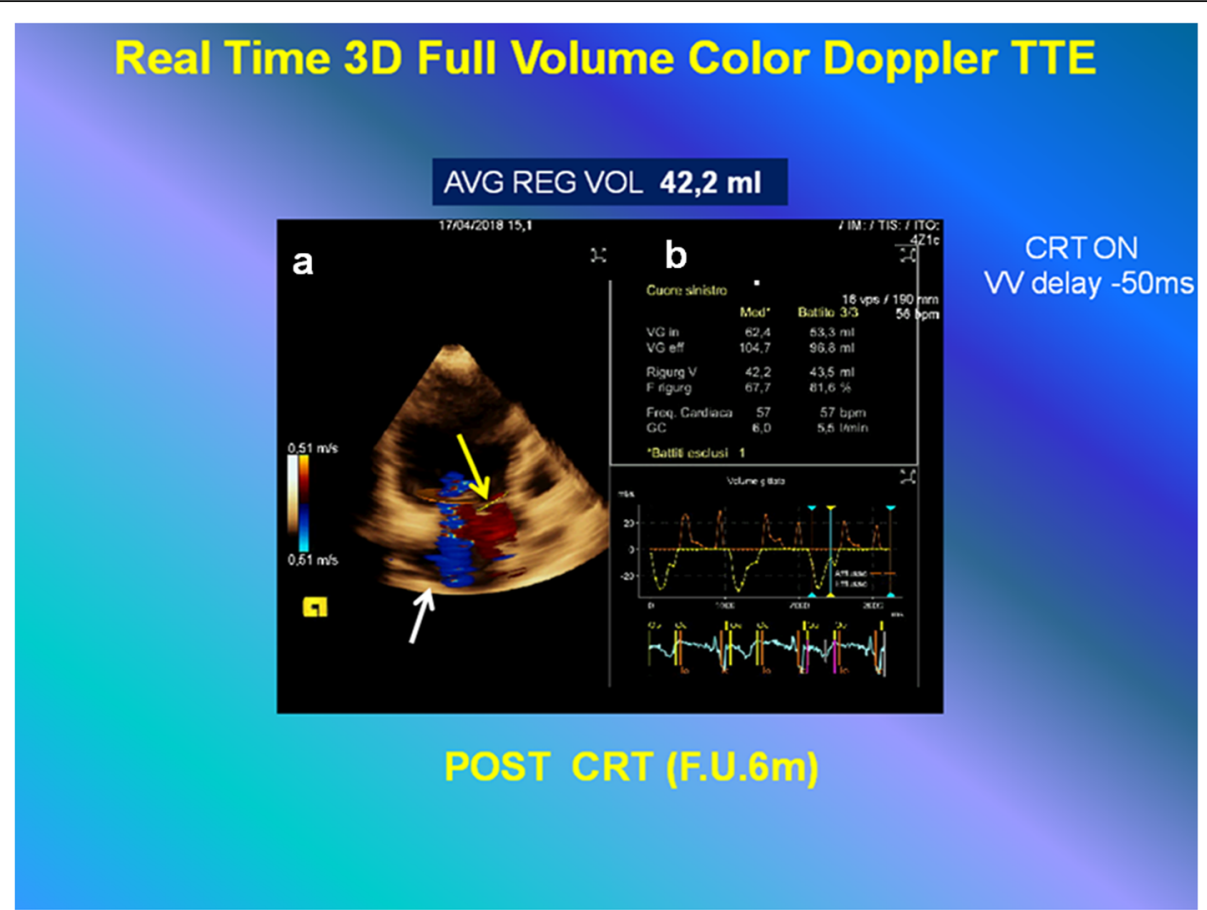

Fig. 6 Quantitative analysis (to compare with the results of analysis represented in Fig. 4), of functional mitral regurgitation by three-dimensional full-volume color Doppler transthoracic echocardiography: at 6-month follow-up setting cardiac resynchronization therapy ON interventricular delay $-50 \mathrm{~ms}$. a Automated anatomy detection of the left ventricular endocardial border, mitral annulus, left ventricular outflow, and placement of three-dimensional hemispheric flow sampling planes in the mitral annulus (white arrow) and left ventricular outflow (yellow arrow). b Flow-time curve derived from automatic flow volume aggregation at each frame in the cardiac cycle and the automated calculation of the mitral (In SV), aortic (Out SV stroke volume, regurgitant volume, regurgitation fraction (RF), and cardiac output (CO), with mean value, in three cardiac cycles, respectively. AVG mean value, CRT cardiac resynchronization therapy, F.U. follow-up, REG VOL regurgitant volume, W interventricular

can radically affect ventricular remodeling with epigenetic nexus [10-12]. LV flow represents an integral outcome of the tissue contraction/relaxation process whose dynamic features (local and short lasting) may not be easily detectable in terms of tissue displacement. The fluid dynamics represents a sort of coupling between systole and diastole without a sharp separation between them [13], and the analysis of flow dynamics inside the LV can provide new information about LV systolic and diastolic function through the analytical representation of the distribution of intraventricular pressure gradients; this is because flow properties at one instant depend on the combination of mechanical events during previous time. The assessment of morphological and energetic characteristics of fluid dynamics, both at baseline pre-implantation and after biventricular pacing, is potentially combinable with three-dimensional FVCD to correct suboptimal device settings. We previously demonstrated that changes in electrical activation alter the orientation of blood flow momentum. The echo-PIV technique may be useful for elucidating the favorable effects of CRT on

Table 1 Profile three-dimensional full-volume Color Doppler of echocardiographic results. The last three rows are the de-aliased mitral inflow, left ventricular outflow, and mitral regurgitation volumes based on the sampled volumetric color Doppler data by three-dimensional full-volume color Doppler transthoracic echocardiography

\begin{tabular}{|c|c|c|c|}
\hline & $\begin{array}{l}\text { Acute study (13-day } \\
\text { follow-up) }\end{array}$ & $\begin{array}{l}\text { Acute study (13-day } \\
\text { follow-up) }\end{array}$ & $\begin{array}{l}\text { Acute study (6-month } \\
\text { follow-up) }\end{array}$ \\
\hline Mean value measure (ml) & CRT OFF & $\begin{array}{l}\text { CRT ON } \\
\text { W delay } 0 \mathrm{~ms}\end{array}$ & $\begin{array}{l}\text { CRT ON } \\
\text { W delay }-50 \mathrm{~ms}\end{array}$ \\
\hline $\begin{array}{l}\text { Three-dimensional full-volume color Doppler } \\
\text { transthoracic echocardiography: mitral inflow volume }\end{array}$ & 100.3 & 83.2 & 62.4 \\
\hline $\begin{array}{l}\text { Three-dimensional full-volume color Doppler transthoracic } \\
\text { echocardiography left ventricular outflow: outflow volume }\end{array}$ & 35 & 21.6 & 104.7 \\
\hline $\begin{array}{l}\text { Three-dimensional full-volume color Doppler transthoracic } \\
\text { echocardiography: mitral regurgitation volume }\end{array}$ & 65.3 & 61.6 & 42.2 \\
\hline
\end{tabular}




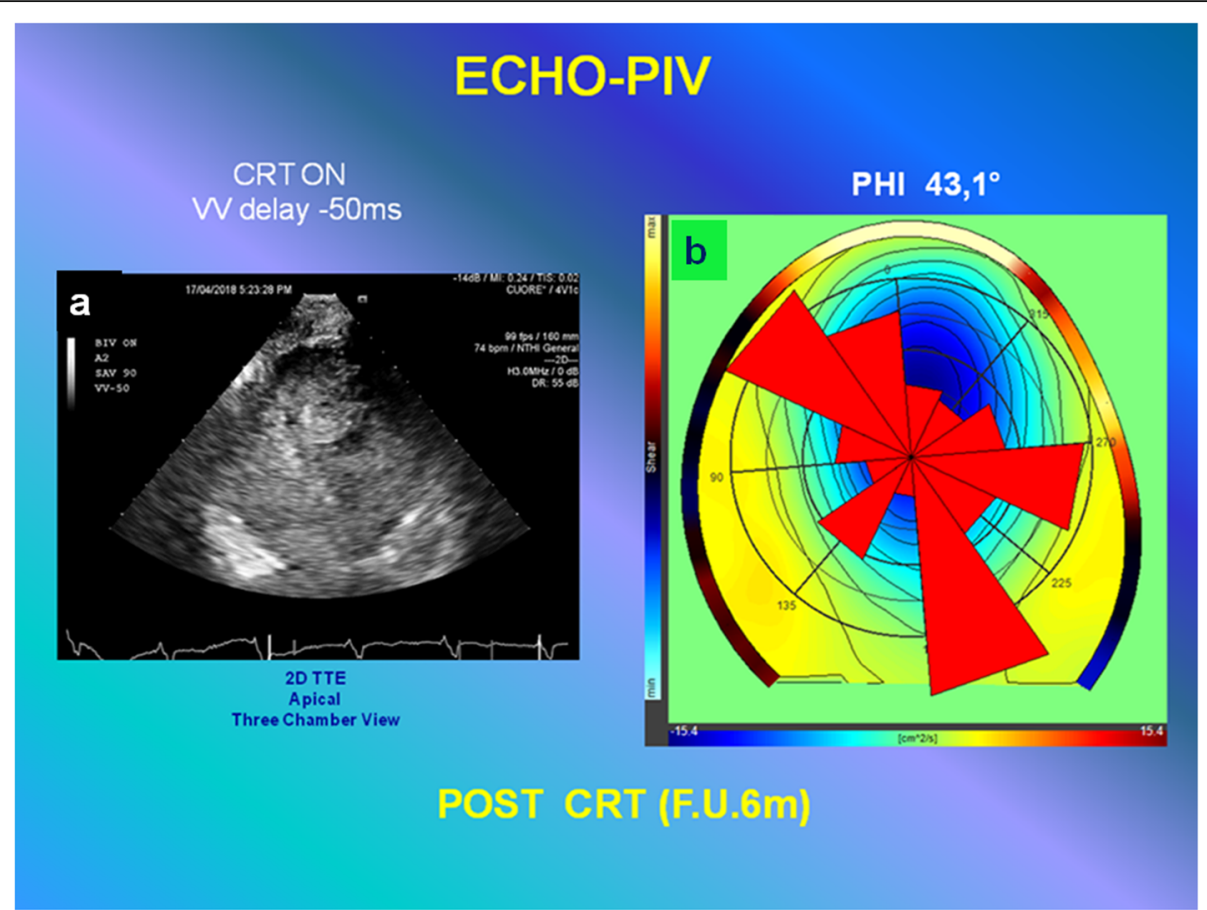

Fig. 7 Quantitative analysis (to compare with the results of analysis represented in Fig. 3), by echo-particle imaging velocimetry of the orientation angle $(\varphi)$ of the global hemodynamic forces exchanged between blood and surrounding tissues during acute study (postcardiac resynchronization therapy 6-month follow-up). a Two-dimensional transthoracic echocardiogram high-temporal resolution contrast echocardiography is performed by three-chamber apical view approach (see Additional file 10: Video S10 interventricular - $50 \mathrm{~ms}$ SONOVUE 6-month follow-up). b The polar histogram showed with setting interventricular delay - 50 ms the most aligned intraventricular forces that were partially dominated by the longitudinal path of pressure gradient (lower $\varphi$, value $43.1^{\circ}$ ). CRT cardiac resynchronization therapy, F.U. follow-up, PIV particle imaging velocimetry, TTE transthoracic echocardiogram, WV interventricular

intraventricular fluid dynamics and it could be used to identify appropriate pacing setting during acute echocardiographic optimization of left pacing vector, with no relevant changes in electrical activation on electrocardiogram, and in PW Doppler mitral inflow or Ao IVT patterns. The long-term CRT outcome correlates with the degree of realignment of hemodynamic forces $[7,8,14]$. The CRT team consisting of experienced electrophysiologists and echocardiologists leads to improved patient outcomes, but current evidence does not strongly support the performance of atrioventricular and ventriculo-ventricular optimization routinely in all patients receiving CRT [15]. The applicability of dyssynchrony optimization in a "real-world" clinical setting is debated [16, 17], although sustained and effective biventricular pacing is crucial to achieving the best outcome from CRT. The degree of realignment of hemodynamic forces, with quantitative analysis of the orientation of blood flow momentum $(\phi)$, can represent improvement of fluid dynamics synchrony of the LV, and explain with a new deterministic parameter the effects of CRT on all the pathophysiologic determinants of FMR through the increase of "closing forces," reduction of the "tethering forces," a reshape of the annular geometry and function, and the correction of diastolic mitral regurgitation, respectively.

\section{Conclusions}

This clinical case offers an innovative and accurate approach for acute echocardiographic optimization of left pacing vector. It shows the clinical utility of combined three-dimensional FVCD/echo-PIV assessment to increase response to CRT, in terms of reduction of FMR, improving fluid dynamics synchrony of the LV.

\section{Additional files}

Additional file 1: Video S1. Transthoracic echocardiogram apical fourchamber view showing a dilated left ventricular pre-implant, with severe functional mitral regurgitation, assessed by qualitative estimation with two-dimensional color flow Doppler approach. (WMV 1610 kb)

Additional file 2: Videos S2. Two-dimensional contrast-enhanced cine loops with a particle image velocimetry technique for different pacing settings: cardiac resynchronization therapy-OFF (Video 2). (WMV 692 kb)

Additional file 3: Video S3. Cardiac resynchronization therapy ON with interventricular delay 0 ms (Video 3). (WMV 4820 kb)

Additional file 4: Videos S4. Cardiac resynchronization therapy ON with interventricular delay - 30 ms (Video 4). (WMV 247 kb) 
Additional file 5: Video S5. Cardiac resynchronization therapy ON with interventricular delay - 50 ms (Video 5). (WMV 645 kb)

Additional file 6: Videos S6. Real-time three-dimensional color flow Doppler quantification at 13-day follow-up, during acute study: cardiac resynchronization therapy OFF (Video 6). (WMV 559 kb)

Additional file 7: Video S7. Cardiac resynchronization therapy ON with interventricular delay 0 ms (Video 7). (WMV 559 kb)

Additional file 8: Video S8. Transthoracic echocardiogram apical fourchamber view, at 6-month follow-up showing a dilated left ventricle post-implant, with moderate-to-severe functional mitral regurgitation, assessed by qualitative estimation with two-dimensional color flow Doppler approach, with a smaller central jet and no reaching of the posterior wall of the left atrium. (WMV $231 \mathrm{~kb}$ )

Additional file 9: Video S9. Real-time three-dimensional color flow Doppler quantification at 6-month follow-up. (WMV 638 kb)

Additional file 10: Video S10. Two-dimensional contrast-enhanced cine loops with a particle image velocimetry technique. (WMV 2920 kb)

\section{Acknowledgements}

None.

\section{Funding}

This research did not receive any specific grant from funding agencies in the public, commercial, or not-for-profit sectors.

\section{Authors' contributions}

$A D, G T, C C, V M C$, and PC: drafting of the manuscript and critical revision of the manuscript for important intellectual content. GP: conception and design of the research, analysis, and interpretation of the data, and supervision. $A R M, V T$, and VB: acquisition, analysis, and interpretation of the data, critical revision of the manuscript for important intellectual content, and supervision. All authors read and approved the final manuscript.

\section{Ethics approval and consent to participate}

This study was performed to conform to the Declaration of Helsinki under the approval of our Institution's Ethical Committee for the protection of human participants.

\section{Consent for publication}

Written informed consent was obtained from the patient for publication of this case report and any accompanying images. A copy of the written consent is available for review by the Editor-in-Chief of this journal.

\section{Competing interests}

The authors declare that they have no competing interests.

\section{Publisher's Note}

Springer Nature remains neutral with regard to jurisdictional claims in published maps and institutional affiliations.

\section{Author details}

${ }^{1}$ Cardiology Division, Monaldi Hospital, AORN Ospedali dei Colli, Via Leonardo Bianchi, 80131 Napoli, Italy. ${ }^{2}$ Cardiology Division, University of Chieti, Chieti, Italy. ${ }^{3}$ Department of Engineering and Architecture, University of Trieste, Trieste, Italy.

Received: 19 December 2018 Accepted: 12 March 2019

Published online: 25 May 2019

\section{References}

1. Thavendiranathan P, Liu S, Datta S, Rajagopalan S, Ryan T, Igo SR, et al. Automated quantification of mitral inflow and aortic outflow stroke volumes by three-dimensional realtime volume color-flow Doppler transthoracic echocardiography: comparison with pulsed-wave Doppler and cardiac magnetic resonance imaging. J Am Soc Echocardiogr. 2012;25:5665. https://doi.org/10.1016/j.echo.2011.10.004.

2. Heo R, Son JW, ó Hartaigh B, Chang HJ, Kim YJ, Datta S, et al. Clinical Implications of Three-Dimensional Real-Time Color Doppler Transthoracic
Echocardiography in Quantifying Mitral Regurgitation: A Comparison with Conventional Two-Dimensional Methods. J Am Soc Echocardiogr. 2017;30: 393-403. https://doi.org/10.1016/j.echo.2016.12.010.

3. Ge S, Bu L, Zhang H, Schelbert E, Disterhoft M, Li X, et al. A real-time 3dimensional digital Doppler method for measurement of flow rate and volume through mitral valve in children: a validation study compared with magnetic resonance imaging. J Am Soc Echocardiogr. 2005;18:1-7. https:// doi.org/10.1016/j.echo.2004.08.021.

4. Gruner C, Herzog B, Bettex D, Felix C, Datta S, Greutmann M, et al. Quantification of Mitral Regurgitation by Real Time Three-Dimensional Color Doppler Flow Echocardiography Pre- and Post-Percutaneous Mitral Valve Repair. Echocardiography. 2015;32(7):1140-6. https://doi.org/10.1111/echo.12809. Epub 2014 Oct 18

5. Kato A, Sandoval JP, Mroczek D, Chaturvedi R, Houle H, Georgescu B, et al. Automated 3-dimensional single beat real-time volume color-flow Doppler echocardiography in children: a validation study of right and left heart flows. Can J Cardiol. 2018;34:726-35. https://doi.org/10.1016/j.cjca.2018.03.001.

6. Zhou X, Thavendiranathan P, Vannan MA. Quantification of Flow by 3D Color Flow Doppler: From Pretty Pictures to Priceless Physiology. Can J Cardiol. 2018;34:703-4. https://doi.org/10.1016/j.cjca.2018.04.028.

7. Pedrizzetti G, Martiniello AR, Bianchi V, D'Onofrio A, Caso P, Tonti G. Changes in electrical activation modify the orientation of left ventricular flow momentum: novel observations using Echocardiographic Particle Image Velocimetry. Eur Heart J Cardiovasc Imaging. 2016;17(2):203-9. https://doi.org/10.1093/ehjci/jev137. Epub 2015 Jun 9

8. Martiniello AR, Pedrizzetti G, Bianchi V, Tonti G, D'Onofrio A, Caso P. Left ventricular pacing vector selection by novel echo-particle imaging velocimetry analysis for optimization of quadripolar cardiac resynchronization device: a case report. J Med Case Rep. 2016;10:191. https://doi.org/10.1186/s13256-016-0965-9.

9. Pedrizzetti G, La Canna G, Alfieri O, Tonti G. The vortex - an early predictor of cardiovascular outcome. Nat Rev Cardiol. 2014;11:545-53. https://doi.org/ 10.1038/nrcardio.2014.75.

10. Pasipoularides A. Mechanotransduction mechanisms for intraventricular diastolic vortex forces and myocardial deformations: Part 1. J Cardiovasc Transl Res. 2015;8(1):76-87. https://doi.org/10.1007/s12265-015-9611-y.

11. Kehat I, Molkentin JD. Molecular pathways underlying cardiac remodeling during pathophysiological stimulation. Circulation. 2010;122:2727-35. https://doi.org/10.1161/CIRCULATIONAHA.110.942268.

12. Marfella R, Di Filippo C, Potenza N, Sardu C, Rizzo MR, Siniscalchi M, et al. Circulating microRNA changes in heart failure patients treated with cardiac resynchronization therapy: responders vs. non-responders. Eur J Heart Fail. 2013;15:1277-88. https://doi.org/10.1093/eurjhf/hft088.

13. Abe H, Caracciolo G, Kheradvar A, Pedrizzetti G, Khandheria BK, Narula J, et al. Contrast echocardiography for assessing left ventricular vortex strenght in heart failure :a prospective cohort study. Eur Heart J Cardiovasc Imaging. 2013;14:1049-60. https://doi.org/10.1093/ehjci/jet049.

14. Siciliano M, Migliore F, Badano L, Bertaglia E, Pedrizzetti G, Cavedon S, et al. Cardiac resynchronization therapy by multipoint pacing improves response of left ventricular mechanics and fluid dynamics: a three-dimensional and particle image velocimetry echo study. Europace. 2017;19(11):1833-40. https://doi.org/10.1093/europace/euw331.

15. Brignole $M$, Auricchio A, Baron-Esquivias $G$, Bordachar P, Boriani G, Breithardt OA, et al. 2013 ESC Guidelines on cardiac pacing and cardiac resynchronization therapy: the Task Force on cardiac pacing and resynchronization therapy of the European Society of Cardiology (ESC). Developed in collaboration with the European Heart Rhythm Association (EHRA). Eur Heart J. 2013;34(29):2281-329. https://doi.org/10.1093/eurheartj/ eht150. Epub 2013 Jun 24

16. Gras D, Gupta MS, Boulogne E, Guzzo L, Abraham WT. Optimization of AV and $W$ delays in the real-world CRT patient population: an International Survey on Current Clinical Practice. PACE. 2009;32:S236-9. https://doi.org/10. 1111/j.1540-8159.2008.02294.x.

17. Gorcsan J III, Abraham T, Agler DA, Bax JJ, Derumeaux G, Grimm RA, et al. Echocardiography for Cardiac Resynchronization Therapy: Recommendations for Performance and Reporting-A Report from the American Society of Echocardiography Dyssynchrony Writing Group Endorsed by the Heart Rhythm Society. J Am Soc Echocardiogr. 2008;21: 191-213. https://doi.org/10.1016/j.echo.2008.01.003. 